\title{
NEOPTERIN AND RECURRENT SPONTANEOUS ABORTION (RSA): THE EFFECT OF CELLULAR IMMUNE SYSTEM ACTIVATION ON SUBSEQUENT PREGNANCY
}

\author{
Songül Ünüvar' ${ }^{1}$, Zübeyde Tanrıverdi ${ }^{2}$
}

\begin{abstract}
:
Background: Recurrent miscarriages are common complications in pregnancy. Neopterin is one of the immunologic biomarkers of several diseases related to activation of the cellular immune system. RSA is associated with immune system related abnormalities.

Objectives: We aimed to investigate the effectiveness of neopterin levels in the early diagnosis of RSA.

Methods: Eighty RSA patients and forty-three healthy controls were included in the study. The neopterin concentrations were determined by the enzyme-linked immunosorbent assay (ELISA) method. For the statistical analysis, Mann-Whitney U test and Pearson correlation test were used the; $p<0.05$ was considered statistically significant.

Results: Serum mean neopterin levels were $16.47 \pm 0.095 \mathrm{nmol} / \mathrm{L}$ in RSA group and $6.14 \pm 0.041 \mathrm{nmol} / \mathrm{L}$ in control group, respectively. Compared to the control group, a statistically significant increase $(p=0.0183)$ in the serum neopterin levels of the patients was observed. There was a negative correlation between serum neopterin level and age in both the control $(\mathrm{R}=0.0774, \mathrm{p}=0.6236)$ and RSA groups $(\mathrm{R}=0.1415, \mathrm{p}=0.2089)$. However, this correlation was not statistically significant $(\mathrm{p}>0.05)$.

Conclusions: With overstimulation of interferon- $\gamma(\mathrm{INF}-\gamma)$ during pregnancy, the production of neopterin increases by monocytes/macrophages. The measurement of neopterin levels in the serum contributes to the early diagnosis of pregnancy losses.
\end{abstract}

UDC Classification: 615.4; DOI: http://dx.doi.org/10.12955/cbup.v5.1065

Keywords: Pregnancy, neopterin, INF- $\gamma$

\section{Introduction}

Two types of pregnancy loss have been described, namely sporadic and recurrent pregnancy loss. Recurrent pregnancy loss is observed at a rate of about $1 \%$, while the rate of sporadic pregnancy loss is reported to be higher (25 to 50\%) and is mostly observed to develop in association with chromosome abnormalities during advanced age pregnancies (Rai \& Regan, 2006). RSA is one of the most common complications of pregnancy and adversely affects many pregnant women (Smith \& Cowchock, 1988).RSA is defined as the occurrence of three or more failed pregnancies between the last day of menses and 20th week of gestation, or fetal rejection less than $500 \mathrm{~g}$ of fetal body weight (Rai \& Regan, 2006; Strobino \& Warburton, 1995; Kwak-Kim et al., 2000).

Factors associated with pregnancy loss include disorders related to genetic, hormonal, metabolic, or uterine anatomy, infections, environmental and occupational exposure (lead, mercury, ethylene, oxide, radiation), and personal habits (alcohol consumption and cigarette smoking), thrombophilia or the immune system (Kwak-Kim et al., 2000; Pandey et al., 2004). Immunological mechanisms are suggested to play a role as a possible explanation for pregnancy losses, in the absence of identification of any of these etiologies (Bellingard et al., 1995).

Neopterin is secreted from T-lymphocytes through the GTP (guanosine triphosphate) cyclohydrolase I enzyme by overexpression of IFN- $\gamma$ from activated monocytes, macrophages, and dendritic cells. The activity of this enzyme is greatly increased by IFN- $\gamma$ and other cytokines (Milich et al., 1995; Fuchs et al., 1992; Muller et al., 1991).

\section{Neopterin Levels in Subsequent Pregnancy}

\section{Study Groups}

A total of eighty women with a history of at least three abortions of unknown etiology which occurred during the first trimester were included in the study. The control group consisted of forty three healthy women. The mean age of the RSA group was $29.11 \pm 0.07$ (year), whereas that of the control group was $31.18 \pm 0.19$ (year).

\footnotetext{
${ }^{1}$ Faculty of Pharmacy, İnönü University, Malatya, Turkey. songul.unuvar@inonu.edu.tr

2 Faculty of Pharmacy, İnönü University, Malatya, Turkey. zubeyde8644@ gmail.com
} 
All individuals in the control group were thoroughly scrutinized about their health status. This study was approved by the Institutional Ethics Committee and conducted in accordance with the principles of the Declaration of Helsinki. A written informed consent was obtained from each participant.

\section{Neopterin Measurement}

Blood samples were collected into $10 \mathrm{cc}$ biochemistry tubes for biochemical and histological tests required for routine follow-up blood sampling. Blood samples were collected into heparin-containing vacuum tubes and centrifuged at $3.500 \mathrm{rpm}$. Supernatants were collected and stored at $-20^{\circ} \mathrm{C}$. Serum neopterin levels were measured using the ELISA (DRG Diagnostics GmbH, Germany) method. Neopterin concentrations were expressed in nmol/L.

\section{Statistical Analysis}

Statistical analysis was performed using the SPSS version 11.50 software. Descriptive data were expressed in mean \pm standard error (SE).The Mann-Whitney $U$ test was used to compare two independent groups, whereas the Pearson correlation test was used to analyze possible relationships between the variables. A $P$ value of $<0.05$ was considered statistically significant.

\section{Discussion}

The elevated serum concentrations of neopterin have been demonstrated in various infections (malaria, measles and septic shock), chronic inflammatory diseases (Crohn disease, ulcerative colitis) and autoimmune disorders (rheumatoid arthritis, thyroiditis), cardiovascular diseases, malignancies and organ transplantations (Muller et al., 1991; Fuchs et al., 1993; Eisenhut, 2013; Yanchun\&Zhidong, 2011). Neopterin is a sensitive indicator of cell-mediated immune activation (Eisenhut, 2013; Yanchun\&Zhidong, 2011; Huber et al., 1984). Neopterin levels can be used as an indicator in the assessment of immunogenic stimulations induced by the fetus and placenta during pregnancy since its production reflects cellular immune response (Munn et al., 1998).

The mean serum neopterin level was $16.47 \pm 0.095 \mathrm{nmol} / \mathrm{L}$ in the women with RSA and6.14 \pm 0.041 $\mathrm{nmol} / \mathrm{L}$ in the control group, indicating statistically significantly higher levels in the women with a history of RSA ( $\mathrm{p}=0.0182)$.

In addition, we found a negative correlation between the serum neopterin levels and age both in the control group $(\mathrm{R}=0.0774, \mathrm{p}=0.6236)$ and in the $\mathrm{RSA}$ group $(\mathrm{R}=0.1415, \mathrm{p}=0.2089)$ (Figure

1).However, this correlation was not statistically significant ( $p>0.05)$.

\begin{tabular}{|l} 
Figure 1. The distribution of neopterin concentrations according to the age. \\
Source: Author
\end{tabular}

A healthy pregnancy is characterized by depression of cell-mediated immune response in addition to an increase in humoral immune response (Kaleli et al., 2005). The production of Th2 cytokines by decidual T-cells contributes to the progress of pregnancy; however, the excessive increase in Th1 response poses a risk for the survival of the fetus. The predominance of Th1 may be associated with pathological conditions such as RSA and preeclampsia (Kaleli et al., 2005; Lin et al., 1993).

Women with recurrent pregnancy losses and/or inadequate implantation have been reported to possess significantly increased peripheral blood Th1 cells compared to normal fertile women. Increased 
proinflammatory cytokines have been suggested to play an important role in recurrent pregnancy losses (Kwak-Kim et al., 2000). Excessive activation of Th1 cytokines and natural killer (NK) cells has been reported to be the most important alloimmune cause of RSA (Pandey et al., 2004). NK cells in the uterine mucosa have been reported to contribute to the cytokine response at the maternal-fetal interface. This cytokine response is generally caused either by type Th1 (interleukin 2, INF- $\gamma$ and tumor necrosis factor alpha (TNF- $\alpha$ ) production) or type Th2 (interleukin 4, 6 and 10) cells. A normal pregnancy may be the result of Th1-type cytokine response. On the other hand, women with a history of RSA present with a predominantly Th1-type response during embryonic implantation and during pregnancy (Rai \& Regan, 2006; Piccinni et al., 1998).

Many studies have been conducted which suggest that complications of pregnancy which lead to disorders of placentation such as preeclampsia and fetal growth retardation are associated with increased decidual cellular immunity (Erkenekli et al., 2015; Bartha \&Comino-Delgado, 1999). About $20 \%$ of pregnancies result in spontaneous abortion. About $60 \%$ of spontaneous abortions are due to genetic, infectious, hormonal and immunological factors. Under certain conditions, the immune tolerance mechanism may be impaired and the fetus immunologically rejected. The effect of immune mechanisms is also associated with the gestational period during which the abortion occurred. During preimplantation and until the end of implantation, cell-mediated immunity is said to be responsible for early abortion. Immunocompetent decidual cells or cytokines have been reported to be responsible for these immunological mechanisms (Giacomucci et al., 1994). The production of IFN- $\gamma$ activates decidual macrophages, causing injury by stimulating the production of nitric oxide and TNF- $\alpha$, which cause apoptosis and inhibit the secretion of granulocyte macrophage colony stimulating factors from the uterine epithelium.Th1 cytokines, which are secreted as a result of IFN- $\gamma$ activity, result in the termination of pregnancy through embryo and trophoblast toxicity. As a result, during RSA, paternal lymphocytes immunotherapy is thought to have a beneficial effect on the provision of specific and non-specific T-cell suppression. The risk of the next pregnancy loss is approximately $24 \%$ in women with a history of two abortions, $30 \%$ after the third, and $40 \%$ after four abortions (Regan et al., 1989).There is a possibility of RSA in women with previous live births. History of previous delivery is an independently associated indicator of the result of the next birth. The risk of abortion increases after each consecutive pregnancy loss and the prognosis worsens with increased maternal age. In advanced age pregnancies, the risk of abortion increases with the decrease in the number and quality of oocyte present. The risk is higher particularly in women over the age of 35 (Green-top Guideline, 2011).

Women with RSA were found to have local and systemic immunological changes when compared to women with normal pregnancy. Implementation of immunological tests to these patients would be beneficial during the appropriate period of pregnancy (Pandey et al., 2004; Magid et al., 1998).

\section{Conclusion}

In conclusion, evaluation of neopterin levels with routine clinical tests during pregnancy would contribute to the prognosis. The measurement of neopterin levels in body fluids using the ELISA method is rare in the clinical practice. The addition of neopterin measurement to routine clinical laboratory practice may contribute to early diagnosis of diseases.

\section{References}

Bartha, J.L.,\&Comino-Delgado, R. (1999). Lymphocyte subpopulations in intrauterine growth retardation in women with or without previous pregnancies. European Journal of Obstetrics and Gynecology,82(1), 23-7.

Bellingard, V., Hedon, B., Eliaou, J.F., Seignalet, J., Clot, J., \&Viala, J.L. (1995). Immunogenetic study of couples with recurrent spontaneous abortions. European Journal of Obstetrics and Gynecology and Reproductive Biology, 60(1), 53-60.

Eisenhut, M. (2013). Neopterin in diagnosis and monitoring of infectious diseases. Journal of Biomarkers, 196432, doi: $10.1155 / 2013 / 196432$.

Erkenekli, K., Keskin, U., Uysal, B., Kurt, Y.G., Sadir, S., Çayci, T., Ergün, A.,Erkaya, S.,Danişman, N.,\&Uygur, D. (2015). Levels of neopterin and C-reactive protein in pregnant women with fetal growth restriction.Journal of Obstetrics and Gynaecology,35(3), 225-8.

Fuchs, D., Weiss, G., Reibnegger, G.,\&Wachter, H. (1992). The role of neopterin as a monitor of cellular immune activation in transplantation, inflammatory, infectious, and malignant diseases. Critical Reviews in Clinical Laboratory Sciences,29(34), 307-41.

Fuchs, D., Weiss, G.,\&Wachter, H. (1993). Neopterin, biochemistry and clinical use as a marker for cellular immune reactions. International Archives of Allergy and Immunology. 101(1), 1-6. 
Giacomucci, E., Bulletti, C., Polli, V., Prefetto, R.A.,\&Flamigni, C. (1994). Immunologically mediated abortion (IMA). The Journal of Steroid Biochemistry and Molecular Biology,49(2-3), 107-21.

Green-top Guideline No. 17 (2011). The Investigation and Treatment of Couples with Recurrent Firsttrimester and Secondtrimester Miscarriage. Royal College of Obstetricians and Gynaecologists.

Huber, C., Batchelor, J.R., Fuchs, D., Hausen, A., Lang, A., Niederwieser, D., Reibnegger, G.,Swetly, P.,Troppmair, J.,\&Wachter, H. (1984). Immune response-associated production of neopterin. Release from macrophages primarily under control of interferon-gamma. The Journal of Experimental Medicine, 160(1), 310-316.

Kaleli, I., Kaleli, B., Demir, M., Yildirim, B., Cevahir, N.,\&Demir, S. (2005). Serum levels of neopterin and interleukin-2 receptor in women with severe preeclampsia. Journal of Clinical Laboratory Analysis, 19(2), 36-9.

Kwak-Kim, J., Kim, J.W.,\&Gilman-Sachs, A. (2000). Immunology and Pregnancy Losses: HLA, Autoantibodies and Cellular Immunity. Madame Curie Bioscience Database (Internet). Austin TX: Landes Bioscience; 2000-2013. Available from: https://www.ncbi.nlm.nih.gov/books/NBK6615/

Lin, H., Mosmann, T.R., Guilbert, L., Tuntipopipat, S.,\&Wegmann, T.G. (1993). Synthesis of T helper 2-type cytokines at the maternal-fetal interface. The Journal of Immunology, 151(9), 4562-73.

Magid, M.S., Kaplan, C., Sammaritano, L.R., Peterson, M., Druzin, M.L.,\&Lockshin, M.D. (1998). Placental pathology in systemic lupus erythematosus: a prospective study. The American Journal of Obstetrics and Gynecology, 179(1), 226-34.

Milich, D.R., Sallberg, M.,\& Maruyama, T. (1995). The humoral immune response in acute and chronic hepatitis B virus infection. Seminars in Immunopathology, 17(2-3), 149-66.

Muller, M.M., Curtius, H.C., Herold, M.,\&Huber, C.H. (1991). Neopterin in clinical practice. Clinica Chimica Acta, 201(12), 1-16.

Munn, D.H., Zhou, M., Attwood, J.T., Bondarev, I., Conway, S.J., Marshall, B., Brown, C.,\&Mellor, A.L. (1998). Prevention of allogeneic fetal rejection by tryptophan catabolism. Science,281(5380), 1191-3.

Pandey, M.K., Thakur, S.,\&Agrawal, S. (2004). Lymphocyte immunotherapy and its probable mechanism in the maintenance of pregnancy in women with recurrent spontaneous abortion.Archives of Gynecology and Obstetrics, 269(3), 161-72.

Piccinni, M.P., Beloni, L., Livi, C., Maggi, E., Scarselli, G.,\&Romagnani, S. (1998). Defective production of both leukemia inhibitory factor and type 2 T-helper cytokines by decidual T cells in unexplained recurrent abortions. Nature Medicine,4(9), $1020-4$.

Rai, R.,\& Regan, L. (2006). Recurrent miscarriage. The Lancet, 368(9535), 601-611.

Regan, L., Braude, P.R.,\&Trembath, P.L. (1989). Influence of past reproductive performance on risk of spontaneous abortion. British Medical Journal, 299(6698), 541-5.

Smith, J.B.,\& Cowchock, F.S. (1988). Immunological studies in recurrent spontaneous abortion: effects of immunization of women with paternal mononuclear cells on lymphocytotoxic and mixed lymphocyte reaction blocking antibodies and correlation with sharing of HLA and pregnancy outcome. JournalofReproductive Immunology, 14(2), 99-113.

Strobino, B.,\& Warburton, D. (1995). Recurrent abortion: genetic and other non immune factors. In: Reed GB, Wairnex AE, Cockburn F (eds) Diseases of the foetus and newborn, pathology, imaging, genetics and management, 2nd ed. Chapman and Hall, London, pp 167.

Yanchun, L.,\&Zhidong, H. (2011). Significance of humoral neopterin in clinical diagnostics and prognosis. Journal of Medical Colleges of PLA, 26(1), 45-51. 